\title{
Les bassins sédimentaires de la mer d'Alboran
}

\author{
par Jean-Marie alzende*, Jean-Piezre REHAGit**, Léo PASTOURET*, Bela SZFP ** \\ et JTAN-LOUIS OLIVE'T*
}

\begin{abstract}
Résumé. I'cxamen de profils sismiques (réalisés au Sparker et au canon à air) effectués en mer d'Alboran nous permet de compléter et modifier une interprétation proposée précédemment. Outre des compléments apporlés au cadre structural, on a pu mettre en évidence l'existence dans les bassins situés de part et d'autre des seuils d'Alboran d'une couche de sel tluant surmontée d'évaporites. D'autre part, il apparait, compte tem de l'épaisse converture infra-salifere reconnue dans le bassin occidental d'Alboran, que le substratum du bassin pris dans son ensemble est à une profondeur identique à celle du bassin nord-africain. Ceci vient à l'appui de l'hypothèse d'une origine et d'unc évolution semblable pour les deux bassins.

A bstract. - The study of seismic profiles (made with Sparker and with air-gun) recorded in the Alboran sea helps us to complete and modify an interpretation which was previously made. Further to complementary data on the structural frame, we showed the existence of a salt laver topped with evaporites, in the basins situated on both sides of the $\Lambda$ lboran ridges. With regard to the thick infra-salt layer in the western Alboran basin, it appears that the basement of the basin, as a whole, is at a depth similar to the depth of the North-African basin. This supports the hypothesis of a common origin and evolution for both basins.
\end{abstract}

\section{Introdiction.}

La mer d'Alboran se différencie topographiquement du bassin nord-algérien qui la prolonge à l'Lst : elle est moins profonde et ses parties basses sont étroites et enserrées entre des zones de forts reliefs (fig. 1). Dans des travaux antérieurs [Olivet et al., 1972, 19731 fondés sur des données encore fragmentaires, nous avions cru pouvoir conclure à l'originalité siructurale de la mer d'Alboran, reflet de ses paricicularilés physiographiques.

En particulier, l'interprétation que nous donnions de l'épisode messinien venait à l'appui de cette idée : la série à sel fluant qui caractérise les parties alyssales de la Míditerranée occidentale n'y avait pas été reconnue. Nous pensions qu'une série évaporitique incomplete s'etait diposée dans un bassin de faible extension à l'list de (iibraltar (bassin occidental d'Alooran) tandis que les marges el les parties moins profondes du bassin avaient été soumises pendant la mème période à une érosion subaćrienre. I)u point de vue structural, cela signifiait que la mer d'Alboran occupait une position " marginale ", surćlevée (comparable à celle du golfe de Valence [Pautot el al., 1972]), par rapport au reste du bassin algéro-provencal. Cette interpretation est partagee plus ou moirs explicitement

B.S.G.F., (7), XVII, 1975, n'1. par les autres auteurs qui ont étudié ce domaine [I.F.P. et C.N.E.X.(O., 1972; Finetti et Morelli, 1973 ; Mulder, 1973] et va dans le sens des interprélations de la gravimétric donnces par S. Coron [1973] et Bonuini el al. [1973]. La mer d'Alboran serait plus "continentale " (moirs "océanisée ") que le bassin algéro-provençal. Sans aborder directement son histoire structurale, nous reprendrons ici le problème de l'évolution sédimentaire néogène de la mer d'Alboran.

Nous avons été conduits, en effet, à compléter et à modifier les interprétations précédentes par l'étude de prolils de sismique reflexion continue (canon a air, Sparker et flexotir) provenant des campagnes Géomède I (1966), Gibraltar 11 (1967) et Géomède II I (1970) du Iaboratoire de geologie dynamique de la Faculté dess sciences de Paris et Polymede II (1972) du Centre ocćanologique de Brelagne (fig. 2). Cette

* Centre océanologique de Bretagne, B.P. 337, 29273 Brest. Contribution no 315 du Département scientifique.

** Station de géodynamique sous-marine, La Darse, 06230 Villefranche-sur-Mer.

L'ordre des auteurs a été tiré au sort. Note déposée le 28 octobre 1974 , présenlée à la séance du 4 novembre 1974 . Manuscrit défnitif reçu le 11 décembre 1974. 


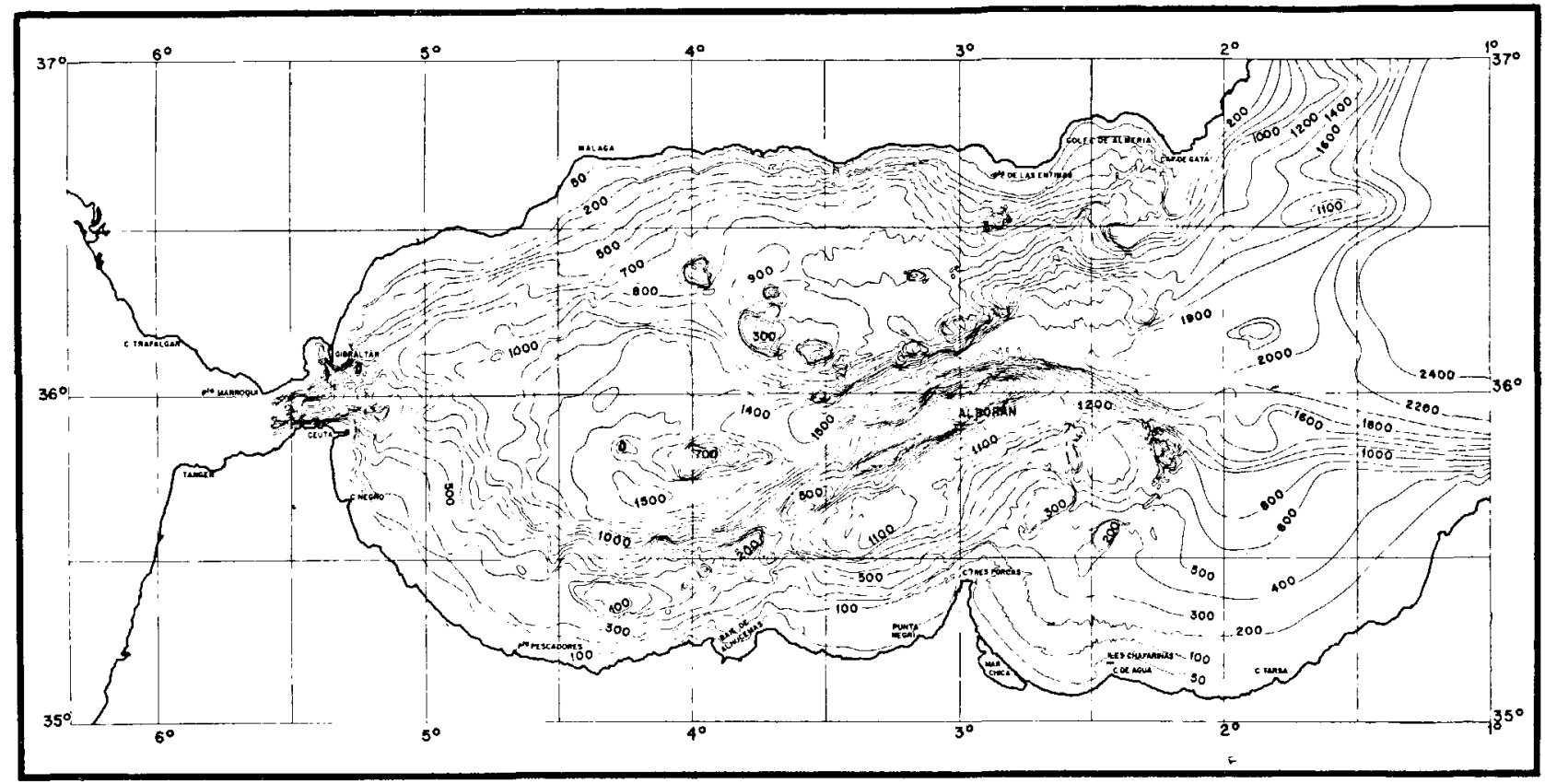

FIG. 1. - Carte bathymétrique d'après des documents du Service hydrographique et océanographique de la marine (entre Gibraltar et $2^{\circ} \mathrm{W}$ ) et de l'Osservatorio di Geofisica Sperimentale (à l'Est de $2^{\circ} \mathrm{W}$ ).

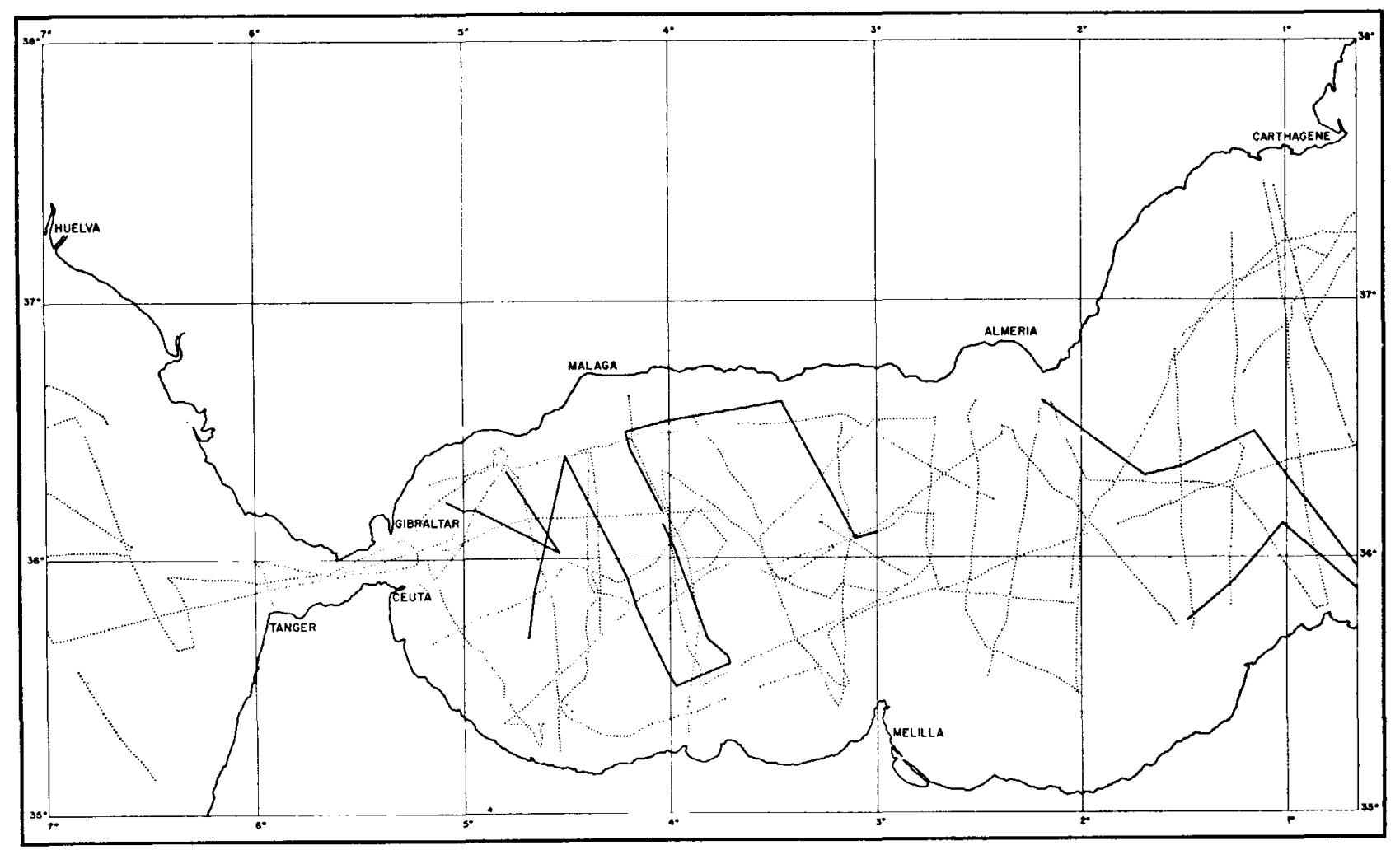

FIG. 2. - Plan de position : en traits pleins : profils sismiques (flexotir) provenant des campagnes Polymède I et II du Centre océanologique de Bretagne. En pointillés : profils sismiques (canon à air, Sparker et Flexotir) provenant des campagnes Géomède I et III et Gibraltar II du Laboratoire de géologie dynamique de la Faculté des sciences de Paris.

B.S.G.F., (7), XVII, 1975, $\mathrm{n}^{\circ} 1$. 
nouvelle étude établit une parenté d'évolution étroite entre le bassin d'Alboran et le bassin nordalgérien.

\section{II. - Le cadife structeral.}

Nous avons représenté sur la figure 3 par une trame foncée les zones hautes du substratum de la mer d'Alboran (zones de profondeur approximativement inférieure à $1500 \mathrm{~m}$ ). Lne trame pointillée indique les zones où le substratum serait compris entre 1500 et $3500 \mathrm{~m}$ de profondeur. Les bassins où le socle est à une profondeur supérieure à $3500 \mathrm{~m}$ sont en blanc, de mème que les bassins néogènes à terre. Nous avons indiqué en noir et avec des rayures les edifices volcaniques respectivement reconnus ou supposés. La détermination de ces derniers s'appuie soit sur leur analogie morphologique avec les volcans connus [Giermann et al., 1968], soit sur les données du magnétisme [Galdeano et al., 1974].
I a disposition des zones surélevées et des bassins fait bien apparaitre les systemes de cassures $\mathrm{XE}-\mathrm{SW}$ et NW-SE que soulignent les alignements des volcans.

On peut observer en particulier dans le secteur occidental la disposition en gradins à regard sudouest du seuil du Djibouti et du seuil qui limite le profond bassin occidental d'Alboran. C"est sur un des massifs appartenant à ce seuil qu'a été effectué le forage 121 du leg XIII du Glomar Challenger. I'existence de ce seuil ainsi que la disposition du bassin qui borde immédiatement au Nord-list la chaine du Rif sont masqués par l'épaisse accumulation des dépòts plio-quaternaires (cf. carte bathymetrique fig. 1 et profil 1, fig. 3 et 4 ). L'n bassin (ou une succession de bassins) bordent la còte espagnole entre Malaga et Almeria.

I.e bassin oriental d'Alboran est moins bien connu. Son extension est moindre et il existe de gros massifs de nature indéterminée dans sa partie centrale. Il

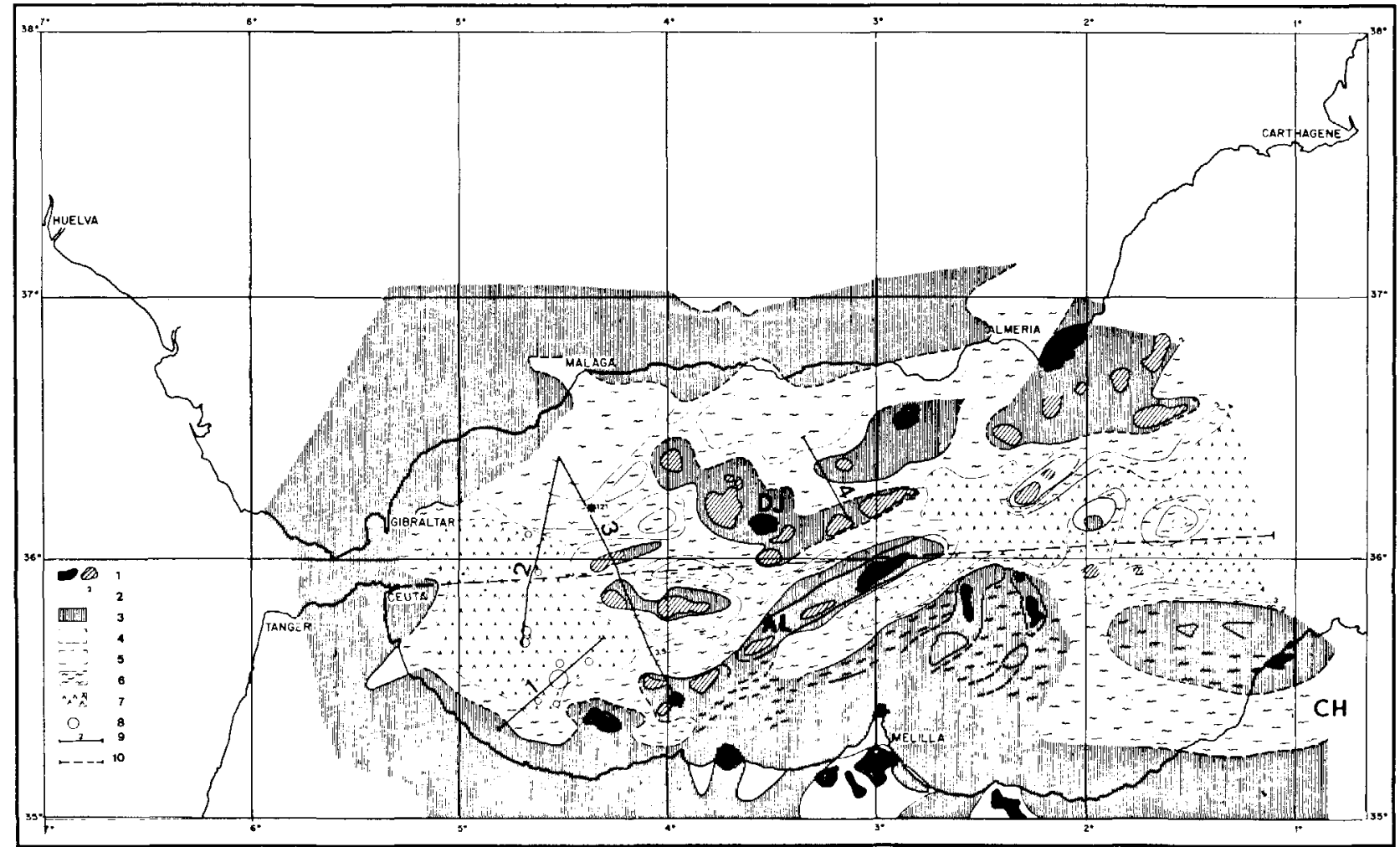

Fia. 3. - Schéma structural de la mer d'Alboran.

1 : volcaus reconnus (en noir) ou supposés (en rayures) ; 2 : isochrones temps double du socle acoustiguc ; 3 : zones où la profondeur du socle esl comprise entre 0 et $1500 \mathrm{~m}: 4$ : zones où la profondeur du socle est comprise entre 1500 et $3500 \mathrm{~m}$; 5: zones où la profondeur du socle est supérieure à $3500 \mathrm{~m} ; 6$ : zones où se sont déposées des évaporites. Nous avons laissé en blanc les bassins néogènes à terre, dans lesquels la série évaporitique n'a pas été décrite ; 7 : zones où s'est déposé le sel fluant (halite) ; 8 : domes de sel ; 9 : position des profils sismiques montrés sur les figures 4 , 6 , 7 et 10; 10 : localisation du profil synthéticue (fig. 8).

DJ : Seuil du Ijjibouti. AL : Seuil d'Alboran. $\mathrm{C}_{4} \mathrm{H}$ : bassin du Chelif.

B.S.G.F., (7), XVII, 1975, n० 1. 


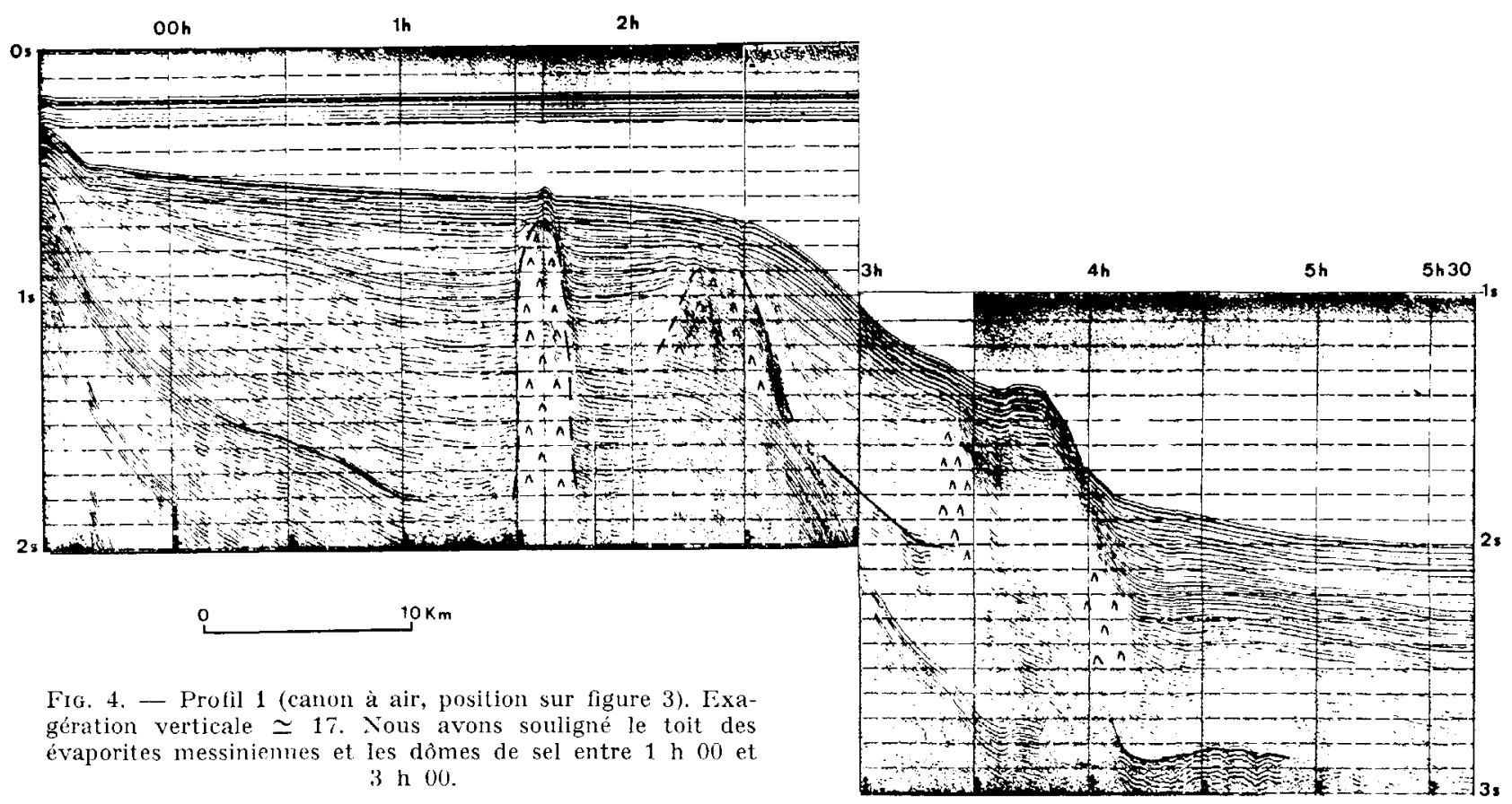

est limité au Sud par une zone haute qui s'étend en regard de l'avant-pays marocain. Quelques données attestent l'existence d'un prolongement immergé du bassin du Chelif à travers cette zone (fig. 3).

I.es données de la carte de l'anomalie gravimétrique à l'air libre établie par Allan et Morelli [1971] (fig. 5) viennent corroborer les traits mis en évidence par la sismique réflexion : en particulier la disposition et l'importance du bassin occidental d'Alboran.

\section{III. - LES BASSINS SÉdIMLNTAIRES.}

Le profil 1 (fig. 4) a été réalisé du Sud-Ouest vers le Nors-Est à travers la marge rifaine et le bassin occidental d'Alboran. Ce profil montre que l'avancée topographique qui existe en regard de la chaine rifaine correspond à une construction sédimentaire récente. I.es structures anticlinales visibles entre $1 \mathrm{H} 30$ et 4 H 00 ont vraisemblablement joué un ròle de barrage; leur morphologie, l'allure des strates à leur contact et l'absence d'anomalies gravimétriques positives nous conduisent à les interpréter comme des diapirs de sel. De part et d'autre de ces structures, les forts réflecteurs situés à $1,8 \mathrm{~s}$ au Sud-Ouest et à $2,8 \mathrm{~s}$ au Nord-Est peuvent représenter le toit d'une série évaporitique surmontant la couche de sel fluant (halite). Comme en mer Ligure [Rehault et al., 1974], le décalage du toit des évaporites de part et d'autre des diapirs suggère le rejeu récent plio-quaternaire d'accidents profonds. Par analogie avec ce que l'on observe dans tout le bassin de la Méditerranée occidentale ainsi qu'au niveau du forage 121 on peut attribuer la série superficielle, régulièrement et nettement stratifiée, et la sćrie sous-jacente, plus " transparente ", au plioQuaternaire ; la série assimilée aux évaporites appartiendrait au Messinien.

Le profil 2 (fig. 6) à travers le bassin occidental d'Alboran montre dans sa partie mediane sous $1.3 \mathrm{~s}$ temps double (soit environ $1300 \mathrm{~m}$ ) de couverlure rapportée au plio-(Muaternaire, un fort réflecteur que nous avious dejà interpreté comme le toit des évaporites messiniennes folivet el al., 1972]. La profondeur de ce réllecteur correspond bien à celle du réflecteur observé sur la partic profonde du profil 1 (fig. 1). Il pourrait s'agir du même réflecteur. Dans le tiers sud-ouest du profil apparaît une série d'ondulations; dans notre première étude [Olivet et al., 1972], nous les avions interprétées soit comme les manifestations d'un socle situé à faible profondeur, soit comme des plissements d'origine tectonique autre que salifere. La première hypothèse n'est en accord ni avec les données gravimétriques (voir ci-dessus fig. 5) ni avec les données magnétiques [Galdeano el al., 1974]. D'autre part, un profil à travers les mèmes structures (cf. [Mulder, 1973], fig. 9) montre indiscutablement que le socle dans celle zone est profondément enfoui (4 à $5 \mathrm{~s}$ temps 


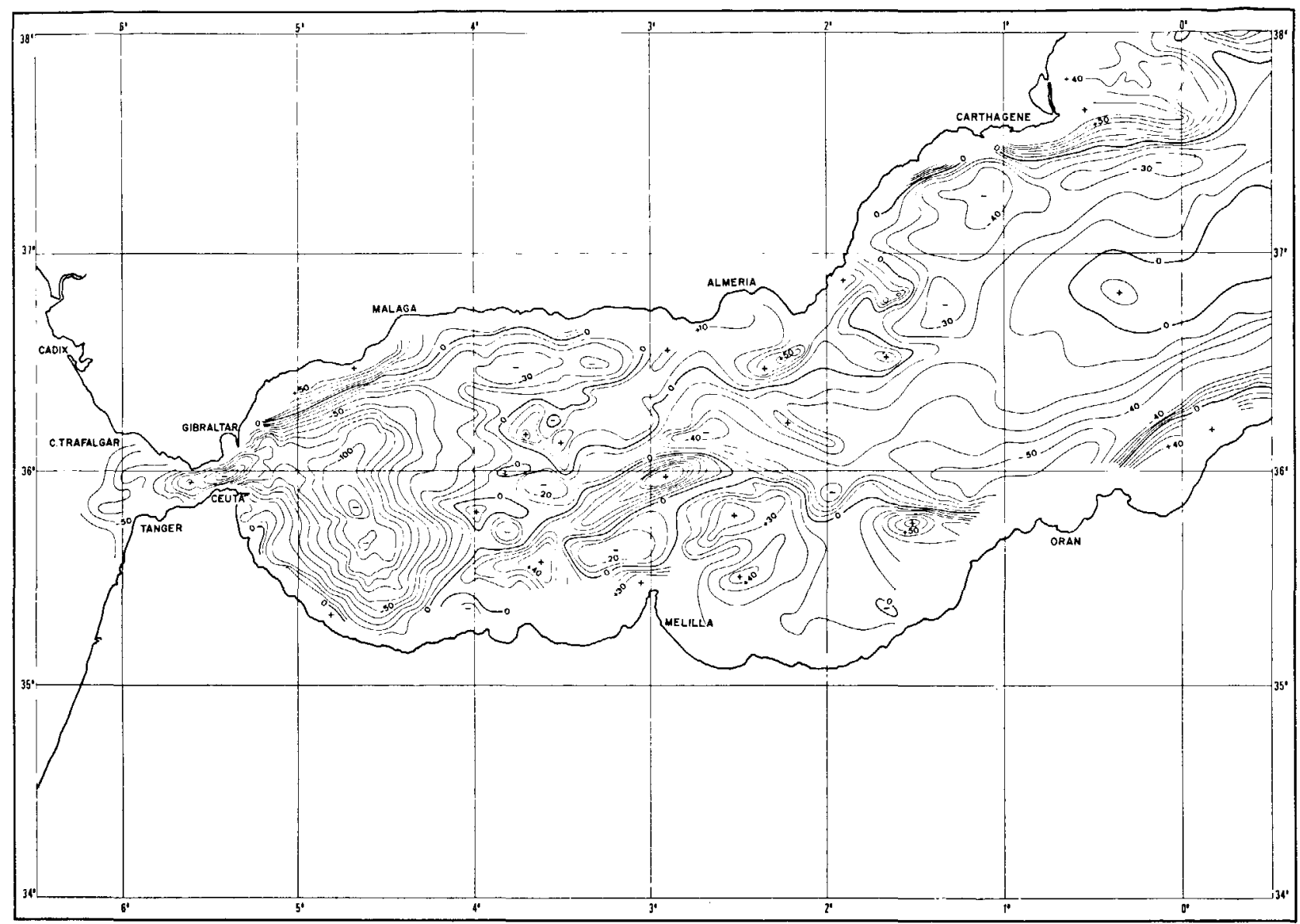

Fig. 5. - Carte de l'anomalie gravimétrique à l'air libre d'après Allan et Morelli [1971].

double au minimum sous le fond). La seconde hypothèse devient peu probable à partir du moment où nous observons par ailleurs (fig. 4) des structures salifères plus caractéristiques. Nous pensons donc que ces ondulations résultent d'une tectonique salifère. Compte tenu de nos observations précédentes, nous pensons que ce sel est d'âge messinien. Mulder [1973] admet également qu'il peut s'agir de dòmes de sel mais il attribue à ce sel un âge Miocène inférieur ou plus ancien sur la base de corrélation entre des profils sismiques réflexion et le forage 121 du DSDP. Pour Mulder, les 1000 à $1500 \mathrm{~m}$ superficiels observables sur les profils sismiques ne comprendraient pas seulement le Plio-Quaternaire, comme nous l'avons proposé, mais également le Miocène supérieur et moyen. Outre le fait qu'une telle réduction d'épaisseur du Plio-Quaternaire dans cette partie du bassin est peu probable et compte tenu de la position du forage 121 (fig. 3) les corrélations qui ont été faites sont sujettes à caution.

Les profils 2 et 3 (fig. 6 et 7 ) permettent de fixer B.S.G.F., (7), XVII, 1975, $\mathrm{n}^{0} 1$. sans ambiguité, à partir du forage 121, l'épaisseur du Plio-Quaternaire sur la marge ibérique et dans le bassin occidental d'Alboran. Le profil 1 (fig. 4) montre une accumulation plus importante le long de la marge rifaine. Ces épaisseurs ( 1 à $1,3 \mathrm{~s}$ temps double, soit 1000 à $1300 \mathrm{~m}$ si l'on adopte une vitesse moyenne de propagation du son de $2 \mathrm{~km} / \mathrm{s} \mathrm{[I.F.P.-}$ C.N.E.X.O., 1972]) sont plus importantes que celles observées dans le bassin nord-algérien, ce qui est logique, compte tenu de leur position respective par rapport aux sources d'apport. Le profil synthétique (fig. 3 et 8 ) schématise ces observations.

Il faut noter que la figure 9 de Mulder [1973, p. 51] ne montre pas nettement, au-dessous des réllecteurs que nous avons interprétés comme le toit des évaporites sur les profils 1 et 2, la succession caractéristique du bassin nord-africain : séquence de forts réflecteurs parallèles d'environ $0,5 \mathrm{~s}$ d'épaisseur correspondant à des évaporites [Alla et al., 1971| - séquence transparente de $0,3 \mathrm{~s}$ d'épaisseur moyenne correspondant au sel fluant (halite) - 


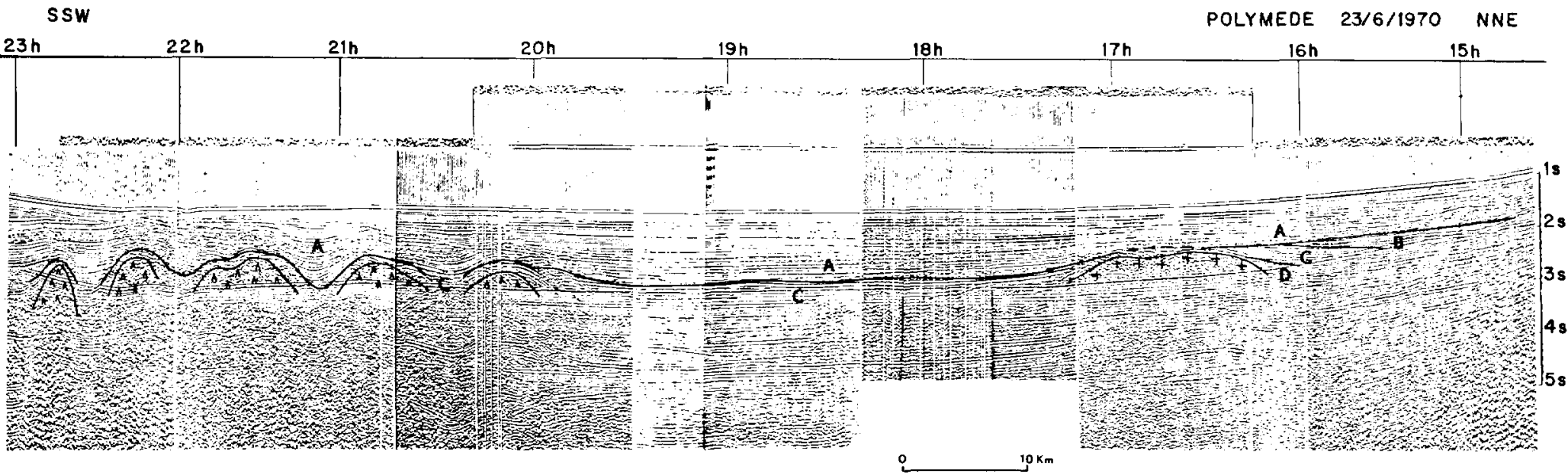

FIG. 6. - Profil 2 (flexotir, position sur figure 3). Exagération verticale $\simeq 5$. Les croix représentent le substratum acoustique.

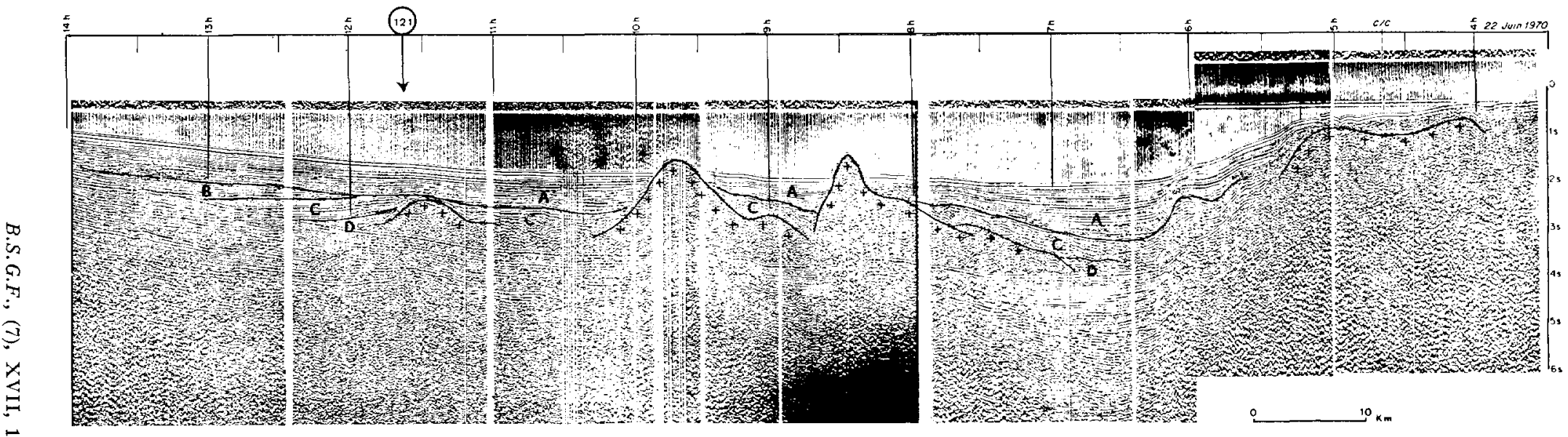

FIG. 7. - Profil 3 (flexotir, position sur fig. 3 ). Exagération verticale $\simeq 5$.

Les croix représentent le substratum acoustique.

A : Plio-quaternaire ; B : Pliocène basal (?) ; C : évaporites ; D : Infra-salifère. 
W.

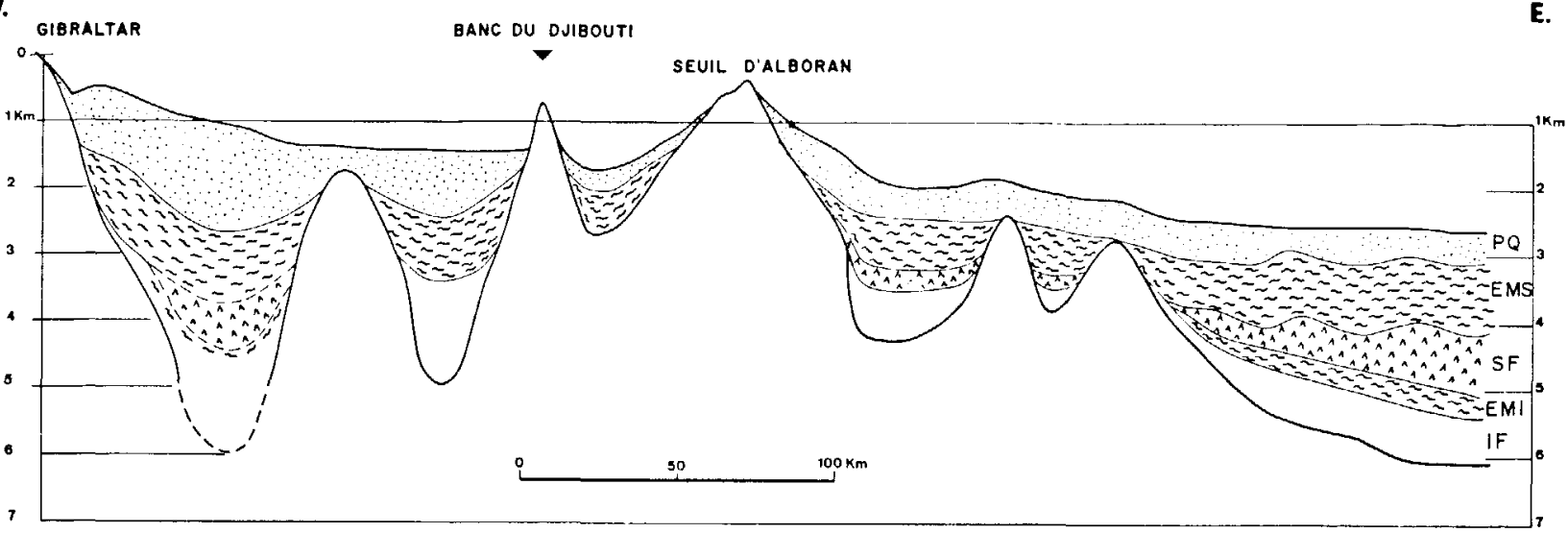

Fig. 8. - Profil synthétique Ouest-Est (position sur figure 3) à travers la mer d'Alboran.

P. Q. : Plio-quaternaire (vilesse : $2 \mathrm{~km} / \mathrm{s}$ ) ; E.M.S. : évaporites messiniennes supérieures (vitesse : $3,7 \mathrm{~km} / \mathrm{s}$ ) ; S. F. : sel fluant (vitesse $: 4,2 \mathrm{~km} / \mathrm{s}$ ) ; E.M. I. : évaporites messiniennes inférieures (vitesse $: 3,7 \mathrm{~km} / \mathrm{s}$ ). I. F. : Infra-salifère (vitesse $3,5 \mathrm{~km} / \mathrm{s}$ ).

et autre séquence de forts réflecteurs pouvant représenter un niveau évaporitique inférieur. Il est possible que dans le bassin d'Alboran, celte succession soit plus complexe sans que cela remette en question la contemporanéité des dépôts de halite dans les deux bassins.

IV. - LE FORAGE 121 ET LES BASSINS DE IAA MARGE ESPAGNOLE.

D'après Ryan el al. [1973], le log du forage 121 est le suivant :

à 16 ) ;

- 670 m de plio-Quaternaire (prélèvements 1

- une surface d'érosion correspondant à l'épisode messinien (entre les prélèvements 16 et 17), l'existence de cette surface étant interprétée à partir des profils sismiques et de l'absence de marqueurs microfaunistiques ;

- $195 \mathrm{~m}$ de sédiments attribués au Tortonien;

- un substratum formé d'éléments métamorphiques de composition voisine de celle du socle bélique et rifain (prélèvement 24 ).

La figure 9 qui est un détail du profil 3 (fig. 7) montre la relation proposée par Ryan et al. [1973] entre le forage 121 et la sismique réflexion.

Nous avions dans un premier temps [Olivet et al., 1972-1973| adopté l'interprétation de l'absence de dépôts messiniens en mer d'Alboran excepté pour un petit hassin à l'Est de Gibraltar où la série évaporitique existait mais sous forme très réduite. Ceci s'accordait avec l'idée que le bassin d'Alboran s'était trouvé au Messinien dans l'ensemble surélevé par rapport au reste du bassin nord-africain, dans une position comparable à celle du golfe du Lion

B.S.G.F., (7), XVII, 1975, no 1 .
[Burrolet et Dufaure, 1972] ou du golfe de Valence [Pautot et al., 1972]. Sur le plan micropaléontologique, cette interprétation a depuis donné lieu à des controverses, portant précisément sur la présence ou l'absence de niveaux évaporitiques messiniens et sur l'âge des sédiments considérés comme tortoniens par Ryan et al. [1973] ou comme Miocène terminal par Montenat, G. et J. J. Bizon (rapport non publié).

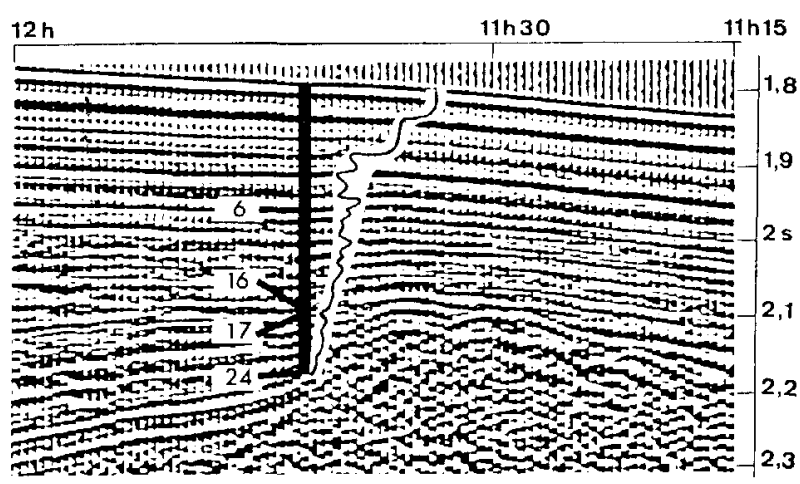

FIG. 9. - Détail du profil 3 (fig. 7) montrant l'interprétation de Ryan et al. [1973] établic à partir des données du forage 121 .

Les éléments nouveaux apportés par les profils de sismique réflexion nous amènent aussi, de notre còté, à modifier cette première interprétation. Nous avons vu qu'il existait dans la partie profonde du bassin occidental d'Alboran une série salifère bien développée ayant donné des dòmes, surmontée par une couche à évaporites, semblables à celle observée dans le reste de la Méditerranée occidentale [Au- 
zende et al., 19711. En bien des points de Méditerranée occidentale [Rehault et al., 1974; Auzende et al., 1971| la série évaporitique supérieure s'étend largement sur les marges. Fn mer d'Alboran, nous avons identifié en particulier dans les bassins qui bordent la marge ibérique au Nord du forage 121 une série de forts réflecteurs qui peuvent ètre interprétés comme évaporiliques. I a description récente de niveaux évaporitiques dans le bassin d'Almeria [Iaccarino et al., 197.1] vient à l'appui de cette identification.

Le forage 121 , s'il est bien placé à l'endroit indiqué sur les figures 7 et 9 , devrait avoir traversé cette série à évaporites. On peut cependant remar- quer que le rapport préliminaire du site 121 [Preliminary report of leg XIII] signale la présence dans les cent derniers mètres du forage (carottes 20 à 24) de cristaux de gypse et de dolomies. L'examen des donnces relatives à la navigation et au positionnement du forage [Preliminary report of leg XIII] nous permet de suggérer qu'il a été effectué à un endroit légèrement différent de celui proposé par Rvan et al., [1973]. Dans ce cas, on peut aisément admettre que le niveau évaporitique, déjà réduit à l'emplacement indiqué (cf. figg. 7) ait été absent.

Si notre interprétation des niveaux à évaporites est correcte (fig. 6, 7 et 10) la discordance bien visible sur les profils ne correspondrait pas à la

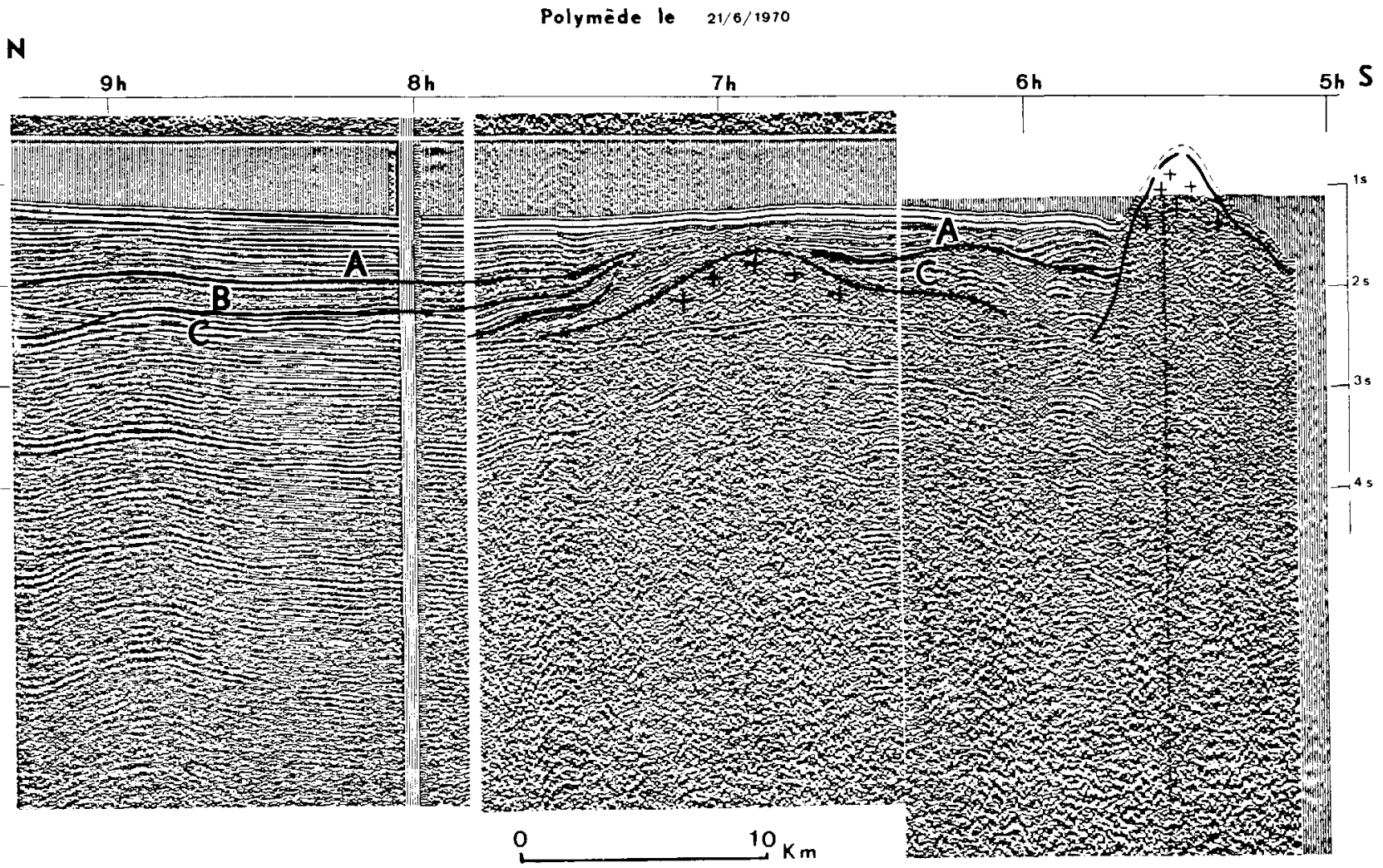

Fig. 10. - Profil 4 (flexotir. Position sur fig. 3). Exagération verticale $\simeq 5$.

A : Plio-Quaternaire ; B : Pliocène basal (?) ; C : évaporites.

Les croix représentent le substratum acoustique.

lacune du Messinien mais à un épisode d'érosion plus tardif, peut-ètre Pliocène inférieur dont la signification reste à déterminer. Il pourrait résuller d'une phase tectonique de soulèvement post-miocène, qui affecterait la marge continentale comme le domaine bétique émergé adjacent Fallot, 1948; Montenat, 1973 ; Bourgeois et al., 1972].

\section{V. - La PARTIE ORIENTALE de LA MER D'Alboran.}

Les profils réalisés entre le bassin nord-africain et la partie orientale de la mer d'Alboran montrent clairement l'extension de la série supérieure à évaporites. La série à sel fluant dont la présence est probable dans les parties les plus profondes n'a pu 
toutefois être mise en évidence de façon certaine.

Les informations encore rares que nous possédons sur la zone haute qui s'ètend au Nord de l'avant-pays marocain nous permettent de suggérer l'existence d'un prolongement immergé du bassin du Chelif et sa communication avec le bassin oriental d'Alboran (fig. 3),

\section{Vi. - Conclusion.}

Dans tout le bassin de la Méditerranée occidentale, les dépôts de sel fluant qui sont à l'origine des dòmes sont confinés aux parties profondes. Ils disparaissent plus ou moins progressivement sur les marges continentales [Auzende et al., 1971 ; Mauffret et al., 1973] et n'existent, de facon distincte sur les enregistrements à une profondeur inférieure à $3,5 \mathrm{~s}$, que dans des secleurs ayant eu une évolution Plio-Quaternaire particulière en raison de leur découplage d'avec les parties profondes du bassin. Ceci a été observé en mer d'Alboran dans la zone qui borde le Rif (fig. 4) où l'horizon donnant naissance aux dômes remonterait jusqu'à moins de $3 \mathrm{~s}$ et en mer Ligure [Rehault et al., 1974]. En général cependant, les zones de la mer d'Alboran où s'est déposée la couche de sel fluant correspondraient aux bassins centraux les plus profonds. Ceux-ci ont dù communiquer constamment entre eux et avec le bassin nord-africain par le détroit d'Alboran. Les évaporites sus-jacentes se sont déposées largement sur les marges et à travers elles sur des domaines maintenant émergés (bassins d'Almeria, du Chelif). Sur le profil schématique de la figure 8 , nous avons restitué les épaisseurs des différentes unités sédimentaires observées sur les profils sismiques réflexion en appliquant les lois de vitesse généralement admises [I.F.P.-C.N.E.X.O., 1972].

(On peut ainsi se rendre compte que la série messinienne se trouve dans le bassin occidental d'Alboran à une profondeur très voisine de celle qu'elle a dans le bassin nord-africain, ainsi que dans le bassin provençal. D'autre part, les épaisseurs totales des sédiments observés en mer d'Alboran (4 à $6 \mathrm{~km}$ selon Mulder [1973]) montrent que la profondeur de son substratum est du même ordre que celle du bassin nord-africain.

Enfin, l'extension latérale du sel fluant et des évaporites que nous avons schématisées sur la figure 3 , montre un modèle très voisin de celui observé par ailleurs en Méditerranée occidentale. Ceci suggère que le bassin d'Alboran et le bassin nord-africain dont les substratums sont à la même profondeur résultent du mème processus de création et ont le même âge. La présence d'une couche salifère et évaporitique permet de penser qu'au moins jusqu'au Messinien les deux bassins ont eu une évolution parallèle.

Remerciements : J. Bonnin et G. A. Auffret ont lu et critiqué le manuscrit. Leurs suggestions nous ont été très utiles.

\section{Bibliographie}

Alila G., Dessolin D. et Leenirardt O. (1971). - Données du sondage sismique continu concernant la sédimentation plio-quaternaire en Méditerranée occidentale. Symp. Méditerranće, Heidelberg (sous presse).

Allan T. D. et Morelli C. (1971), - A geophysical study of the Mediterranean. Bol. Geof. Tenr. Appl., 13 , p. 99-142.

Auzende J. M., Bonnin J., Olivet J. L., Pautot G. et Mauffret $\Lambda$. (1971). - Upper Miocene salt layer in the western Mediterranean Basin. Nature Phys. Sci., 230, p. $82-84$.

Bonini W. E., Loomis T. P. et Robrrtson J. D. (1973). Gravitiy anomalies ultramafic intrusions and the tectonics of the region around the Strait of Gibraltar, J. geophys. Res., 78, p. 1372-1382.

Bourgois J., Cinave P. et Peyre Y. (1972)..- I.es phénomènes tectono-sédimentaires dans l'ouest des Cordillères bétiques depuis l'Arruitanien. Ann. Sci. Univ. Besançon, 3e sér., p. 97-112.
Burrolet P. F. et Dufaure P. (1972). - The Neogene series drilled by the well Mistral $n^{\circ} 1$ in the Gulf of Lion. In, Stanley D. J. et al. The Mediterranean sea. Dowden Hutchson and Ross. Inc, p. 91-98.

Coron S. (1973). -- Grandes zones d'anomalies de la pesanteur dans le bassin méditerranéen et ses bordures. Détails pour la région de Gibraltar. Rapp. P. V. Réun. C.I.E.S.M. 22, 2 a, p. 31-33.

Faliot P. (1948). - Les Cordillères bétiques. Estud. Géol, 8 , p. 83-172.

Finetti I. et Morelli C. (1973). - Geophysical exploration of the Mediterranean sea. Boll. Geof. Teor. Appl., $\mathrm{XV}, 60, \mathrm{p} .263-340$.

Galdeano A., Gouktillot V., Le Borgne F., I. Mouel J. C. et Rossignol J. C. (1974). - Aeromagnetic survey of south-western Mediterranean. Earth and Planet. Sci. Letters (sous presse).

Gifrmane G., Pfannenstiel M. et Wimmenauer W. (1968). - Relations entre morphologie, tectorique et volcanisme en mer d'Alboran (Méditerranée occi-

B.S.G.F., (7), XVII, 1975, no 1 . 


\section{BASSINS DE LA MER D'ALBORAN}

dentale). Résultats préliminaires de la campagne J. Chareot (1967). C.R. somm. S.G.F., p. 116-118

Iaccarivo S., Mohlotti E., Papaxi G., Pelosio G. et RAFFI S. (1974), - Litostratigrafia e biostratigrafia di alcune serie neogeniche della provincia di Almeria (en préparation).

INSTITUT FRANCAIS DU PÉTROLE (I.F.P.) - C.N.E.X.O. (1972). - Données actuelles sur l'histoire tertiaire et la structure de la Méditerranće. Rapport non public, $110 \mathrm{p}$.

Mauffret A., Fall J. P., Montadert L., Sancho J. et Wrixock E. (1973). - Northwestern Mediterrancan sedimentary basin from seismic reflection profile. Am. Ass. Petrol. Geol. Bull, 57, 11 p., p. 2245-2262.

Montenat C. (1973). - I es formations néogenes du Levant espagnol. Thise, Orsay, $1167 \mathrm{p}$

Mulder C. J. (1973). - Tectonic framework and distribution of Miocene evaporites in the Mediterranean. In Messinian events in the Mediterranean, 1973. Kon. Ned. Ak, van Wetenschappen, Amsterdan, p. 41-59.
Olivet J. L., Pautot G. et Auzende J. M. (1972). - Alboran sea : structural framework. In Initial Reports of the Deep Sea Drilling Project, vol. 13, Washington (C.S. Government printing (office), 48-1, p. 1417-1430.

Olivet J. L., Auzenue J. M. et Bonnin J. (1973). — Structure et évolution tectonique du bassin d'Alboran. Bull. Soc. géol. Fr., 7, XV, 2, p. 108-112.

Pautot G., Auzende J. M., Olivet J. L. et Mavffret A. (19-2). - Valencia basin. In Initial Reports of the Deep Sea Drilling Project, vol. 13, Washington (U.S. Government printing Office, 48-2, p. 1430-1441.

PRELIMINAKY REPORT OF LEg XIII (D.S.D.P.). Manuscrit non publié.

IRehault J. P., Olivet J. I. et Auzendf. J. M. (1974). L.e bassin nord-occidental méditerrancen : structure et évolution. Bull. Soc. géol. Fr., (7), XVI, p. 281-294.

Ryan W. B. F., Hsu K. I. et al. (1973), - - Initial Reports of the Decp Sea Drilling Project, vol. 13, Washington, (U.S. Government printing Ollice), p. 43-89. 